\title{
INFLUÊNCIA DA HIPERTENSÃO NA SOBREVIDA DO ENXERTO RENAL EM PACIENTES PEDIÁTRICOS
}

\author{
Samantha Santiago Nagasako, Paulo Cesar Koch Nogueira*, Paula G. P. Machado, José O. Medina Pestana \\ Trabalho realizado no Hospital do Rim e Hipertensão, São Paulo, SP
}

\author{
*Correspondência: \\ Rua Pageu 100 apto 11 , \\ Chácara Inglesa \\ CEP 04139-000 \\ São Paulo-SP \\ pckoch@uol.com.br
}

\begin{abstract}
RESUMO
OBjetivo. Avaliar a influência da hipertensão arterial sistêmica com um ano de transplante renal na sobrevida do enxerto renal três anos após o transplante em crianças.

Métodos. Estudo observacional e retrospectivo na série de pacientes transplantados renais pediátricos da Universidade Federal de São Paulo (UNIFESP) no período de janeiro/I998 a janeiro/2003. Ao final do primeiro ano pós-transplante, os pacientes foram classificados em dois grupos: normotensos e hipertensos. A análise estatística de sobrevida foi através do método de Kaplan-Meier. A comparação entre grupos foi realizada utilizando-se o teste do "log-rank". Para os testes adotamos o limite de 5\% $(\alpha \leq 0,05)$ para rejeição da hipótese de nulidade.
\end{abstract}

Resultados. Antes do transplante 86 pacientes (64\%) e após um ano 70 indivíduos (52\%) foram classificados como hipertensos, respectivamente. A sobrevida do enxerto renal após três anos de transplante foi de $92,5 \%$ para a amostra completa do estudo. O grupo de normotensos apresentou sobrevida de $95,3 \%$ e os hipertensos $90 \%$; a diferença não foi estatisticamente significante.

Conclusão. Apesar do resultado estatístico não ser significante, a diferença observada entre os dois grupos após três anos de transplante, de 5\% maior sobrevida nos indivíduos que eram normotensos um ano após o transplante, nos parece clinicamente significativa e nos permite levantar a hipótese de que a hipertensão arterial pode ser um fator de risco para a sobrevida do enxerto pediátrico. Entretanto, não nos seria possível afirmar que a hipertensão é fator de risco independente para menor sobrevida do enxerto devido às limitações do estudo.

UnITERMOS: Transplante renal pediátrico. Hipertensão. Sobrevida do enxerto.

\section{INTRODUÇÃO}

Atualmente, as principais causas de perda de enxerto renal a longo prazo são a nefropatia crônica e o óbito do paciente; hipertensão arterial é fator associado na gênese de ambos. Tanto a hipertensão prétransplante quanto a pós-transplante são fatores de risco para nefropatia crônica!.

Diversos estudos em adultos mostraram haver correlação entre hipertensão arterial pós-transplante e desenvolvimento de nefropatia crônica do enxerto, sugerindo que a hipertensão não seja apenas uma conseqüência da deterioração da função renal nesses pacientes, mas sim um fator de risco para a nefropatia crônica do enxerto ${ }^{2-7}$.

Em crianças também já foi demonstrada a correlação negativa entre hipertensão arterial sistêmica pós-transplante e função do enxerto renal-12. No entanto, são escassos os estudos que avaliaram o impacto da hipertensão arterial na sobrevida do enxerto renal.

O objetivo do presente estudo é avaliar a influência da hipertensão arterial sistêmica com um ano de transplante renal na sobrevida do enxerto renal três anos após o transplante em crianças.

\section{Métodos}

Foi realizado estudo observacional e retrospectivo na série de pacientes transplantados renais pediátricos (definidos como indivíduos até 18 anos no momento do transplante) em transplantes realizados na Universidade Federal de São Paulo (UNIFESP) no período de janeiro//998 a janeiro/2003.

Ao final do primeiro ano pós-transplante, os pacientes foram classificados em dois grupos: normotensos e hipertensos. A hipertensão arterial foi definida como uso de medicação anti-hipertensiva ou pelo menos três medidas de pressão arterial com valores acima do percentil 95, para pressão sistólica e/ou diastólica, para sexo, idade e estatura de acordo com o Fourth Report on the Diagnosis, Evaluation, and Treatment of High Blood Pressure in Children and Adolescents' ${ }^{13}$.

Considerou-se hipertensão prévia ao transplante a utilização de medicação hipotensora.

Foram considerados como fatores de exclusão do estudo os pacientes com prontuário incompleto, com perda do enxerto ou óbito antes do primeiro ano após o transplante renal e presença de gestação. 
Tabela I - Dados demográficos e nefrológicos dos pacientes, classificados de acordo com o diagnóstico de hipertensão arterial

\begin{tabular}{lccc}
\hline Variável & Controles $(\mathbf{n}=\mathbf{6 4})$ & Casos $(\mathbf{n}=\mathbf{7 0})$ & $\mathbf{p}$ \\
\hline Idade do receptor (anos) & $12,5 \pm 4.1$ & $12.9 \pm 3,8$ & NS \\
Idade do doador (anos) & $27,3 \pm 15,4$ & $25,7 \pm 16,7$ & NS \\
Tempo em diálise (meses) & $23,4 \pm 23,5$ & $28,8 \pm 52,0$ & NS \\
Receptor do sexo masculino & $38(59 \%)$ & $40(57 \%)$ & NS \\
Transplante com doador cadáver & $30(47 \%)$ & $40(57 \%)$ & NS \\
Ocorrência de rejeição aguda confirmada por biópsia & $17(27 \%)$ & $21(30 \%)$ & NS \\
Ocorrência de isquemia fría > 24 horas & $0(0 \%)$ & $9(13 \%)$ & 0,030 \\
Ocorrência de retardo da função do enxerto & $14(22 \%)$ & $27(39 \%)$ & 0,036 \\
Glomerulopatia com doença de base & $15(23 \%)$ & $25(36 \%)$ & NS \\
Transsplante preemptivo & $7(11 \%)$ & $3(4 \%)$ & NS \\
\hline
\end{tabular}

Para análise estatística dos dados procedemos a construção de curvas de sobrevida utilizando o método de Kaplan-Meier. A comparação entre grupos foi realizada utilizando-se o teste do "log-rank". Para todos os testes adotamos o limite de $5 \%(\alpha \leq 0,05)$ para rejeição da hipótese de nulidade.

\section{Resultados}

No período estudado foram realizados $|6|$ transplantes renais pediátricos no nosso serviço e 134 foram incluídos no estudo. As causas de não-inclusão foram: gestação (Quatro casos) e seguimento inferior a um ano (23 casos). Dentre as causas de perda por menos de um ano de seguimento tivemos: trombose de artéria renal (Sete), glomerulonefrite de novo (Três), perda de seguimento (Quatro), rejeição aguda (Três), nefropatia crônica do enxerto (Dois), recidiva da doença de base (Dois) e óbito (Dois).

A média de idade dos receptores no transplante renal foi de 12,7 anos (2,2- 18 anos). O peso médio dos pacientes no transplante era de 35,2 quilos (I 0,5-83 quilos). O tempo médio de diálise dos pacientes foi de 26,2 meses (0-42 meses). A média de idade dos doadores foi de 26,5 anos (2-67 anos). O tempo médio de isquemia fria foi de 567,6 minutos (19- 1860 minutos). Em 75 pacientes (56\%), a etiologia da insuficiência renal foi glomerulopatia ou indeterminada. Quanto ao tipo de transplante, 70 casos eram com doador cadáver (52,2\%) e 64 com doador vivo $(47,8 \%)$.

Hipertensão arterial prévia ao transplante ocorreu em 86 pacientes (64\%); desses, 55 permaneceram hipertensos e 31 experimentaram normalização da pressão arterial. Por outro lado, dos 48 casos que eram normotensos antes do transplante, 33 permaneceram assim e 15 desenvolveram hipertensão após um ano. Dessa forma, ao final do primeiro ano de transplante, 70 pacientes (52\%) eram hipertensos enquanto 64 estavam normotensos. Os dois grupos foram semelhantes entre si em relação aos dados demográicos e nefrológicos dos pacientes, exceto pelo tempo de isquemia fria e retardo de função do enxerto (Tabela I).

A sobrevida do enxerto renal após três anos de transplante foi de $92,5 \%$ para a amostra completa do estudo. O grupo de normotensos apresentou sobrevida de 95,3\% e o grupo de hipertensos, 90\%; essa diferença não foi estatisticamente significante (Figura I).

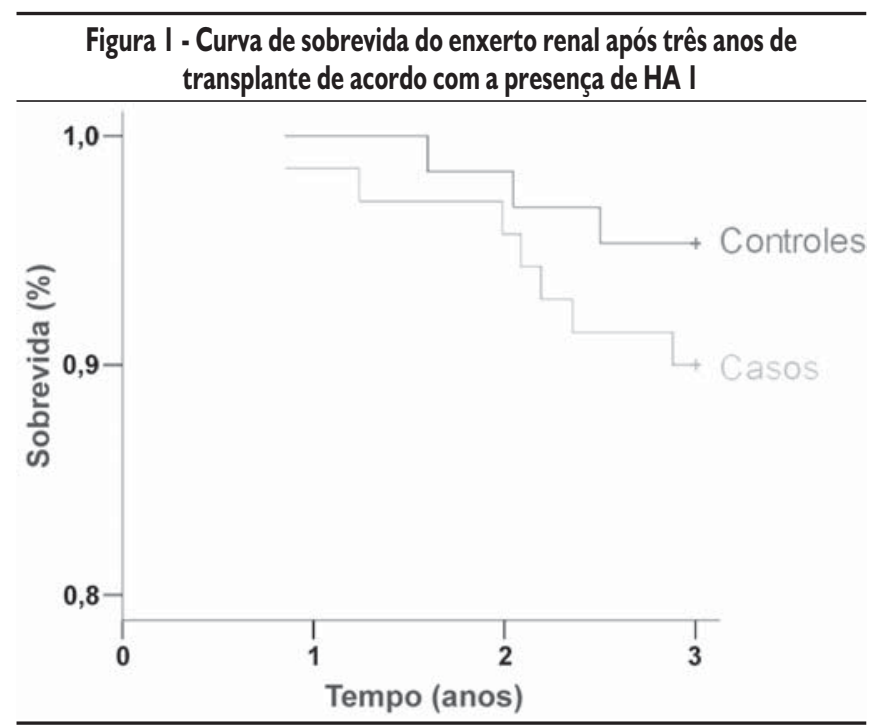

\section{Discussão}

A hipertensão arterial sistêmica é uma complicação freqüente no transplante renal, com prevalência de até $80 \%$ em algumas séries estudadas e representa um importante fator de risco para doença cardiovascular e menor sobrevida do enxerto renal tanto em adultos quanto em crianças8, 9, 11, 12, 14-17.

Nosso estudo mostrou-se de acordo com a literatura em relação à prevalência de hipertensão pós-transplante renal. Baluarte et al. ${ }^{18}$ encontraram prevalência de $70 \%$ no primeiro mês pós-transplante e de $59 \%$ no segundo ano. Em outra série demonstrou-se $60 \%$ de crianças hipertensas com seis meses de transplante renal, 56,6\% com um ano e $46,4 \%$ no terceiro ano pós-transplante ${ }^{19}$. Sorof et al..$^{8}$ observaram prevalência de hipertensão arterial em $58 \%$ das crianças com cinco anos de tranplante renal. Mitsnefes et al. ${ }^{12}$, em $62 \%$ das crianças com mais de um ano de tranplante renal.

Quanto à função e à sobrevida do enxerto renal, há estudos demonstrando pior função e menor sobrevida para os pacientes 
hipertensos em relação aos normotensos. Chew-Wong, ${ }^{7}$ em uma série de 215 transplantes em adultos, encontrou sobrevida do enxerto renal estatisticamente menor nos pacientes hipertensos ( 51 meses versus 60 meses para os normotensos). Kokado observou sobrevida de $89,5 \%$ e $57,9 \%$ com dois e cinco anos de transplante para os pacientes hipertensos e $100 \%$ e $92,3 \%$ para os normotensos, respectivamente.

Sorof et al. ${ }^{8}$, em estudo colaborativo com 4.383 crianças, concluíram que 0 uso de medicação anti-hipertensiva em crianças transplantadas renais é fator de risco independente para disfunção tardia do enxerto. O mesmo autor, em outro estudo, agora com amostra de 85 crianças transplantadas renais, demonstrou que as crianças hipertensas possuíam pior função do enxerto renal'.

Wigger et al. ${ }^{10}$, em estudo sobre a influência da hipertensão arterial na taxa de filtração glomerular em período de cinco anos de seguimento, também encontraram resultado desfavorável para crianças submetidas a transplante renal e com hipertensão durante 0 seguimento. Em estudo publicado anteriormente a respeito da hipertensão no sexto mês pós-transplante renal pediátrico ${ }^{20}$, nós também observamos pior taxa de filtração glomerular para os pacientes hipertensos em relação aos normotensos (70 vs $\left.83 \mathrm{ml} / \mathrm{min} / \mathrm{l}, 73 \mathrm{~m}^{2}\right)$.

Mitsnefes et al. ${ }^{12}$, em estudo com 217 transplantes renais pediátricos, demonstraram risco dobrado de disfunção do enxerto a cada 10\% de elevação dos níveis de pressão arterial sistólica no primeiro ano de transplante.

O presente estudo mostrou que a hipertensão antes do transplante tende a permanecer assim após um ano, destacando a importância da hipertensão prévia como fator de risco para hipertensão pós-transplante renal, conforme demonstrado na literatura $2,7,17,21,22$. Além disso, nosso estudo mostrou tendência de menor sobrevida do enxerto renal nos pacientes que são hipertensos após um ano de transplante, porém sem diferença estatisticamente significante entre os grupos.

É possível fazer a hipótese que a não-demonstração de diferença estatisticamente significante em nosso estudo tenha ocorrido pela reduzida amostra, o que diminui o poder do estudo para enxergar uma diferença que realmente existe (erro $\beta$ ). De fato, utilizando-se os dados da atual casuística em programa para o cálculo do poder do estudo para encontrar diferenças entre sobrevidas de dois grupos, observamos que nosso estudo com a dimensão da casuística apresentada tem poder muito baixo, girando em torno de 7\%, para discriminar tal diferença. Isso ocorre porque a magnitude do afastamento das duas curvas, da ordem de $5 \%$, exigiria um estudo com efetivo muito grande para determinar se tal diferença ocorre por acaso ou realmente pela ocorrência da hipertensão $0^{23}$.

A nosso ver esse achado não deve ser creditado apenas à hipertensão antes do tranplante, uma vez que em nossa amostra mais de um terço das crianças que eram hipertensas antes do transplante evolveram com normalização de pressão. Além disso, ao repetirmos a comparação de sobrevidas de enxerto três anos após o transplante, considerando a hipertensão antes do transplante como fator de risco, observamos que a deferença entre os hipertensos e os normotensos caiu para apenas $2 \%$ ( $92 \%$ vs $94 \%$, respectivamente). Esses dados favorecem nossa hipótese de que a pressão elevada após o transplante pode ser um fator de risco para a sobrevida do enxerto.
Um ponto crítico que deve ser levado em conta na interpretação de nossos dados é que existem outros fatores além da hipertensão arterial que potencialmente podem ter afetado a sobrevida do enxerto nos grupos estudados. Assim, a ocorrência de tempo de isquemia fria superior a 24 horas e também a ocorrência de retardo na função do enxerto foram significantemente mais freqüentes no grupo de pacientes que cursou com hipertensão do que nos controles. Quando analisamos cada um desses fatores isoladamente, porém, observamos que nenhum deles interferiu na sobrevida dos enxertos de maneira significante. A sobrevida de três anos dos enxertos dos pacientes que não tiveram isquemia fria superior a 24 horas foi de $93 \%$ comparada a $89 \%$ daqueles que apresentaram tal fator. Por outro lado, a sobrevida dos enxertos dos pacientes que apresentaram retardo na função do enxerto foi de $98 \%$, comparada a $89 \%$ dos indivíduos que não tiveram essa complicação. Esse último resultado inclusive é paradoxal, uma vez que a ocorrência de retardo na função do enxerto deveria produzir menor sobrevida e não foi o que observamos. Portanto, dos dois potenciais fatores de confusão de nosso estudo, somente a ocorrência de isquemia fria maior do que 24 horas deveria ser considerada como fonte de viés na interpretação de nossos dados.

Do ponto de vista prático, a diferença observada em nossos dois grupos após três anos de transplante, de $5 \%$ maior sobrevida nos indivíduos que eram normotensos um ano após o transplante, nos parece clinicamente significativa e nos permite levantar a hipótese de que a hipertensão arterial pode ser um fator de risco para menor sobrevida do enxerto pediátrico. Não nos seria possível afirmar se esse fator é independente de outros pela limitação devido à reduzida amostra e à natureza observacional de nosso estudo, já abordadas. No entanto, acreditamos que essa hipótese mereceria ser testada em estudos multicêntricos e, se confirmada, reforçaria a necessidade de buscarmos precocemente a melhor prevenção, diagnóstico e mais eficiente tratamento da hipertensão pós-transplante, não apenas pelas comorbidades ligadas à hipertensão como também para preservar a função do enxerto renal a longo prazo.

\section{Conflito de interesse: não há}

\section{SUMMARY}

INFLUENCE OF HYPERTENSION ON RENAL ALLOGRAFT SURVIVAL IN PEDIATRIC PATIENTS

BACKGROUND. To evaluate the effect of I year systemic arterial hypertension on 3-year allograft survival in children with kidney transplantation.

METHODS. A retrospective study was carried out of pediatric patients submitted to kidney transplantation at the Universidade Federal de São Paulo (UNIFESP) between January, 1998 and January, 2003. Patients were classified as normotensive or hypertensive according to presence of hypertension within the first year after transplantation. Survival analyses were performed with the Kaplan-Meier survival method, and survival curves were compared with the log-rank test. A p value of $<0.05$ was considered statistically significant.

RESULTS. Prior to transplantation there were 86 patients (64\%) and after I year, 70 children (52\%) were classified as hypertensive, 
respectively. Overall, the 3-year graftsurvival was of $92.5 \%$. Survival of the normotensive group was $95.3 \%$ and $90.0 \%$ for the hypertensive group; the difference was not statistically significant.

CONCLUSION. Although the difference between the two groups was not statistically significant the higher survival of the normotensive group seems to be clinically significant and allows hypothesizing that arterial hypertension could be a risk factor for pediatric graft survival. However, due to limitations of the study it is impossible to affirm that hypertension is an independent risk factor for lower graft survival. [Rev Assoc Med Bras 2008; 54(5): 396-9]

KEY WORDS: Pediatric kidney transplantation. Hypertension. Graft survival.

\section{REFERÊNCIAS}

I. Midtvedt K, Neumayer HH. Management strategies for posttransplant hypertension. Transplantation. 2000;70(Suppl I I):SS64-9.

2. Sánchez J, Pallardó LM, Sánchez P, Garcia J, Orero E, Beneyto I, et al. Risk factors and prognostic significance of hypertension after renal transplantation. Transplant Proc. 1992;24(6):2738-9.

3. Kokado Y, Takahara S, Kameoka H, Okuyama A. Hypertension in renal transplant recipients and its effect on long-term renal allograft survival. Transplant Proc. 1996;28(3): 1600-2.

4. Cosio FG, Falkenhain ME, Pesavento TE, Henry ML, Elkhammas EA, Davies EA, et al. Relationships between arterial hypertension and renal allograft survival in african-american patients. Am J Kidney Dis. 1997:29(3):419-27.

5. Mange KC, Cizman B, Joffe M, Feldman HI. Arterial hypertension and renal allograft survival. JAMA. 2000;283(5):633-38.

6. Kasiske BL, Anjum S, Shah R, Skogen J, Kandaswamy C, Danielson B, et al. Hypertension after kidney transplantation. Am J Kidney Dis. 2004;43(6): | 07|-81

7. Chew-Wong A, Alberú-Gómez J, Abasta-Jiménez M, Márquez-Díaz F, Correa-Rotter R. Hipertensión arterial postrasplante renal: factores de riesgo asociados e influencia en la supervivencia del injerto renal. Gac Méd Méx. 2005; | 4|(6):46|-7.

8. Sorof JM, Sullivan EK, Tejani A, Portman RJ. Antihypertensive medication and renal allograft failure: a North America Pediatric Renal Transplant Cooperative Study Report. J Am Soc Nephrol: 1999; I ( I 0): I 324-30.

9. Sorof JM, Goldstein SL, Brewer ED, Steiger HM, Portman RJ. Use of antihypertensive medications and post-transplant renal allograft function in children. Pediatr Transplant. 2000;4(I):21-7.
10. Wigger M, Druckler E, Muscheites J, Stolpe HJ. Course of glomerular filtration rate after renal transplantation and the influence of hypertension. Clin Nephrol. 200 I;56(Suppl I):S30-4.

II. Mitsnefes MM, Omoloja A, McEnery PT. Short-term pediatric renal transplant survival: blood pressure and allograft function. Pediatr Transplant. 200 I;5(3): 160-5.

12. Mitsnefes MM, Khoury PR, McEnery PT. Early posttransplantation hypertension and poor long-term renal allograft survival in pediatric patients. J Pediatr. 2003; | 43( I):98- 103.

13. National High Blood Pressure Education Program Working Group on High Blood Pressure in Children and Adolescents. The Fourth Report on the Diagnosis, Evaluation, and Treatment of High Blood Pressure in Children and Adolescents. Pediatrics. 2004: I | 4(2):555-76.

14. Luke RG, Curtis JJ, Jones P, Whelchel JD, Diethelm AG. Mechanisms of posttransplant hypertension. Am J Kidney Dis. 1985;5( I):A79-84.

15. Curtis JJ. Hypertension and kidney transplantation. Am J Kidney Dis. 1986;7(3): | 8|-96.

16. Curtis JJ. Hypertension following kidney tranplantation. Am J Kidney Dis. 1994:23(3):47| -5.

17. Budde K, Waiser J, Fritsche L, Zitzmann J, Schreiber M, Kunz R, et al. Hypertension in patients after renal transplantation. Transplant Proc. | 997;29(I-2):209-II.

18. Baluarte HJ, Gruskin AB, Ingelfinger JR, Stablein D, Tejani A. Analysis of hypertension in children post renal transplantation- a report of the North American Pediatric Renal Transplant Cooperative Study (NAPRTCS). Pediatr Nephrol. 1994;8(5):570-3.

19. Fontana I, Verrina E, Basile G, Arcuri V, Pellicci R, Tommasi GV et al. Arterial Hypertension in the Transplanted Child: Frequency and Analysis of the Causes. Transplant Proc. 1994;26(1):79-80.

20. Nagasako SS, Nogueira PCK, Machado PGP, Pestana JOM. Arterial hypertension following renal transplantation in children-a short term study. Pediatr Nephrol. 2003; | 8( | 2): | 270-4.

21. Nagasako SS, Nogueira PCK, Machado PGP, Pestana JOM. Risk factors for hypertension 3 years after renal transplantation in children. Pediatr Nephrol. 2007;22(9): | 363-8.

22. Ducloux D, Motte G, Kribs M, Abdelfatah AB, Bresson-Vautrin C, Rebibou JM et al. Hypertension in renal transplantation: donor and recipient risk factors. Clin Nephrol. 2002;57(6):409- I3.

23. DupontWD, PlummerWD. Power and sample size calculations. A review and computer program. Control Clin Trials. 1990; I (2): I 16-28.

Artigo recebido: 03/05/07

Aceito para publicação: 26/03/08 